\title{
Facile preparation of polymer nanotube using self-assemblied metal nanotube as sacrificial template
}

\author{
Lei WANG ${ }^{1}$, Yuxin $\mathrm{GUO}^{1}$, Xianjun $\mathrm{CAO}^{1}$, Gonghao $\mathrm{LU}^{1, \mathrm{a}}$, Xue $\mathrm{LI}^{2, \mathrm{~b}}$ \\ ${ }^{1}$ School of Chemical Engineering, University of Science and Technology Liaoning, Anshan, 114051, \\ China \\ ${ }^{2}$ School of Material and Metallurgy, University of Science and Technology Liaoning, Anshan, \\ 114051, China \\ aemail: ghlu@ustl.edu.cn, bemail: xli@ustl.edu.cn
}

Keywords: Polymer nanotube; Template; Metal organic nanotube; Self-assembly

\begin{abstract}
Polymer nanotube (PNT) is a polymer nanomaterial with tubular structure. In this study, we have developed a simple technique for the fabrication of polymer nanotubes by using self-assembled metal organic nanotube (MONT) as a sacrificial template and dendritic amine and acid as precursor molecules. MONT was firstly prepared by the self-assembly of amphiphilic molecule 1 with copper(II) nitrate in methanol. And then, a dendritic amine [tris(2-aminoethyl) amine, tris-amine] was added to a dispersion of MONT in THF. A coating layer of the tris-amine formed on the surface of MONT. The coating layer on the nanotube surface was further cross-linked by an active ester of citric acid. Finally, the self-assemblied template was easily removed in hot ethanol and the PNTs were obtained by filtration. The results showed that the cross-linked polymers form PNTs up to $80 \%$ when the amount of the tris-amine is 0.4 molar

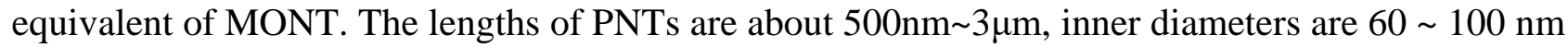
and outer diameters are $80 \sim 120 \mathrm{~nm}$.
\end{abstract}

\section{Introduction}

Polymer nanotubes (PNTs) have attracted considerable attention due to their diverse applications, ranging from controlled drug delivery to biotechnologies [1-5]. Template method is an important and effective method for preparation of special structural and functional nanomaterials[6-8]. By using template method, the unique structure, morphology and function of materials can be controlled by the expected design, and good template is the key to preparation of special functional materials [9-13].

Self-assembled metal organic nanotube (MONT) is a hollow tubular material, prepared by self-assembly of amphiphilic molecules and metal ions [14]. MONT is a good template potential for the preparation of PNT because of simple preparation process and cheap raw materials. Shimizu group reported several MONT systems prepared by adding metal ions into the self-assembling system of amphiphilic molecules with different carbon chains [15-18]. The MONTs with various metal ions on the surface were successfully obtained. They subsequently reported the preparations of $\mathrm{CuO}$ and $\mathrm{Mn}_{2} \mathrm{O}_{3}$ nanotubes using MONTs covered with copper ions as a template [19]. Since the interactions among self-assembly molecules are weak, the structures of MONTs ere easily able to be destroyed at high temperature, strong acid, alkali condition, or even in some organic solvents. Therefore, the preparations of PNTs using MONTs as the template have an practical significance.

In this paper, we will report a simple process for the preparation of PNT by using MONT as a template and dendritic amine and acid as the precursor molecules.

\section{Results and discussion}

Synthesis of the amphiphilic molecule 1 [19]: As shown in Scheme 1, a solution of EDC $\cdot \mathrm{HCl}$ in $\mathrm{CHCl}_{3}$ was added a solution of myristic acid and $\mathrm{HOSu}$ in $\mathrm{CHCl}_{3}$ with stirring at $-10{ }^{\circ} \mathrm{C}$. After the mixture was stirred at $0{ }^{\circ} \mathrm{C}$ for $1 \mathrm{~h}$, a solution of ethyl glycylglycinate hydrochloride in $\mathrm{CHCl}_{3}$ and 
triethylamine was added to the reaction mixture. The mixture was stirred for another $1 \mathrm{~h}$ at $0{ }^{\circ} \mathrm{C}$ and overnight at room temperature. The reaction mixture was washed with $10 \%$ citric acid, $4 \%$ $\mathrm{NaHCO}_{3}$, brine and water, dried over anhydrous $\mathrm{MgSO}_{4}$, filtered and evaporated under reduced pressure to give a white powder. The powder product was subjected to alkali hydrolysis and acidification in ethanol to give a white precipitate. The white precipitate was filtered, washed and dried in vacuum. The amphiphilic molecule 1 was obtained as a white powder in $93.6 \%$ yield. m.p. $168.4 \sim 170.0^{\circ} \mathrm{C} ;{ }^{1} \mathrm{H}$ NMR (500 MHz, DMSO- $\left.d_{6}\right) \delta 12.59(\mathrm{~s}, 1 \mathrm{H}), 8.07$ (s, $\left.1 \mathrm{H}\right), 8.03(\mathrm{~s}, 1 \mathrm{H})$, 3.86 3.59 (m, 4H), 2.12 (s, 2H), 1.49 (s, 2H), $1.25(\mathrm{~s}, 20 \mathrm{H}), 0.86(\mathrm{~s}, 3 \mathrm{H}) ;{ }^{13} \mathrm{C}$ NMR (125 MHz, DMSO- $\left.d_{6}\right) \delta 172.98,171.59,169.86,42.21,41.12$, 40.47, 40.30, 35.67,31.76, 29.53, 29.50, 29.48, 29.40, 29.29, 29.17, 25.57, 22.55, 14.40; IR (kBr) v: 3291, 3082, 2915, 1725, 1608,1559, 1462, $1420,1274,720 \mathrm{~cm}^{-1} ;[\mathrm{M}+1]=343.3$.

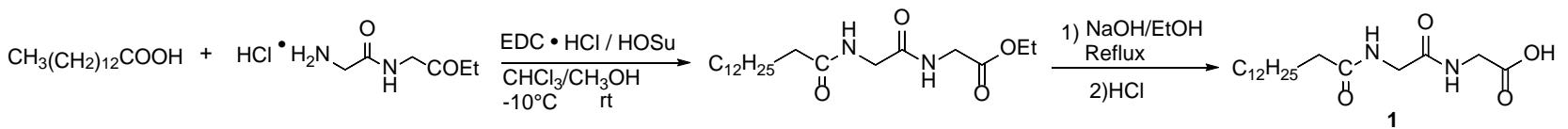

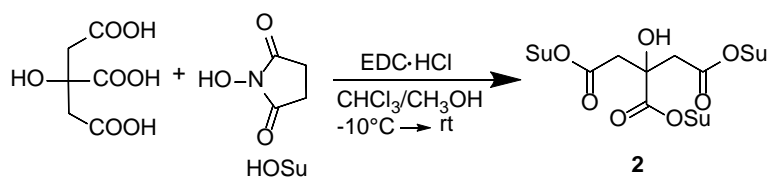

Scheme 1. Preparations of amphiphilic molecule $\mathbf{1}$ and active ester $\mathbf{2}$

Synthesis of active ester of citric acid 2 [20]: As shown in Scheme 1, a solution of EDC $\cdot \mathrm{HCl}$ in $\mathrm{CHCl}_{3}$ was dropped into a solution of citric acid and $\mathrm{HOSu}$ in $\mathrm{CHCl}_{3} / \mathrm{CH}_{3} \mathrm{OH}$ at $-10{ }^{\circ} \mathrm{C}$. After addition, the mixture was stirred for $1 \mathrm{~h}$ at $0{ }^{\circ} \mathrm{C}$ and overnight at room temperature. The reaction mixture was washed with $10 \%$ citric acid, $4 \% \mathrm{NaHCO}_{3}$, brine, and water. The organic layer was dried over anhydrous $\mathrm{MgSO}_{4}$, filtered and evaporated under reduced pressure to give colorless oil in $25.6 \%$ yield.

Preparation of MONT: MONT was prepared by the self-assembly of $\mathbf{1}$ with $\mathrm{Cu}\left(\mathrm{NO}_{3}\right)_{2}$ in methanol/water at room temperature [19]. The tubular structures were confirmed by scanning electron microscope (SEM) and scanning transmission electron microscopy (STEM), as shown in Fig. 1. The self-assemblied MONTs have the lengths from hundreds of nanometers to several micrometers and most of tubes have the lengths of $800 \mathrm{~nm} \sim 3 \mu \mathrm{m}$. Outer diameters are $60 \sim 100 \mathrm{~nm}$, inner diameters are $20 \sim 30 \mathrm{~nm}$ and wall thicknesses are $20 \sim 40 \mathrm{~nm}$.
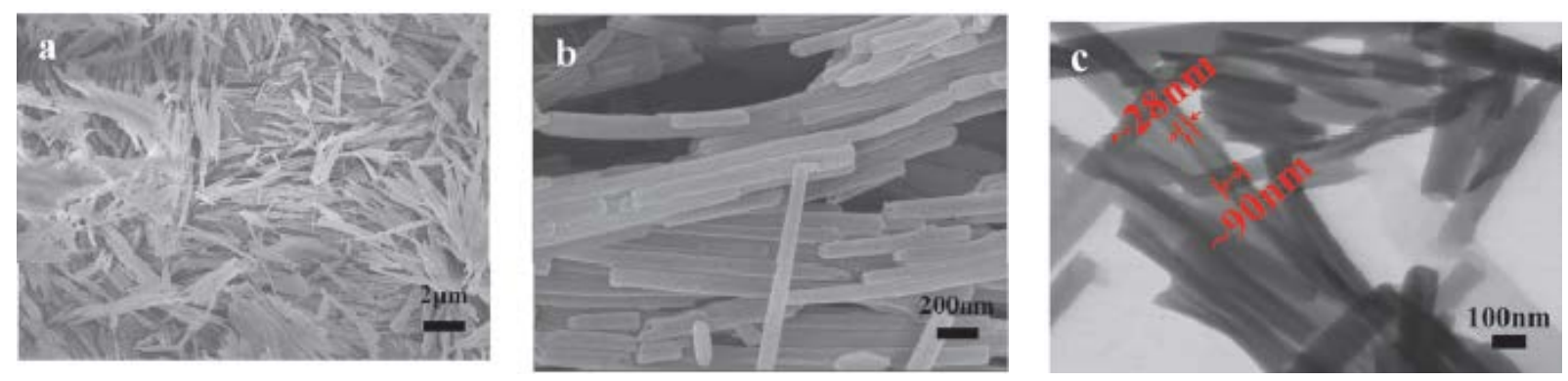

Fig. 1. SEM (a, b) and STEM (c) images of MONT

Preparation of PNT: Since $\mathrm{Cu}^{2+}$ as a transition metal ion has good coordination ability, the MONT covered with $\mathrm{Cu}^{2+}$ on the surface is a potential template by forming $\mathrm{Cu}(\mathrm{II})$-complex. Dendritic tris(2-aminoethyl) amine (tris-amine) formed a complex layer with $\mathrm{Cu}^{2+}$ ions on the surface of MONT, and then a cross-linked reaction was carried out by a coupling reaction with the dendritic active ester $\mathbf{2}$ to form a stable polymer layer on MONT surface. Finally, PNTs were obtained by dissolving the self-assemblied template in hot ethanol and subsequent heat filtration. The preparation process for PNT is shown in Fig. 2. 


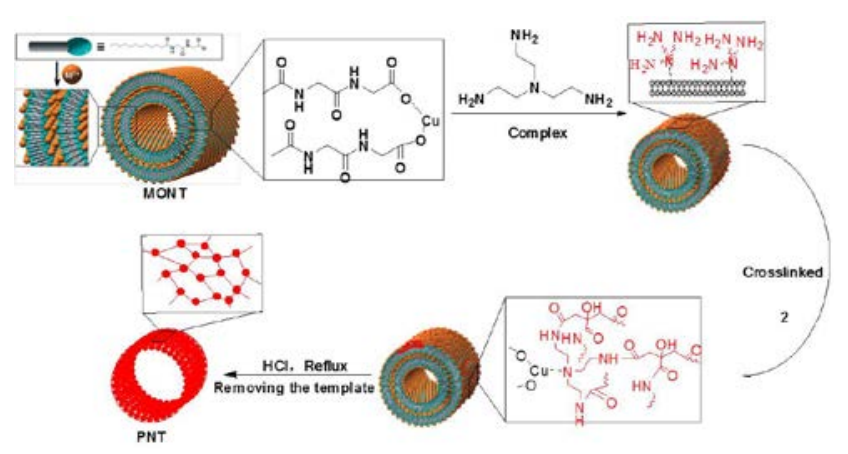

Fig. 2. Schematic process for PNT preparation
Table 1. Molar ratio of tris-amine and active ester 2 to MONT $(0.2 \mathrm{mmol})$

\begin{tabular}{ccccc}
\hline Enter & $\begin{array}{c}\text { Tris- } \\
\text { amine }\end{array}$ & $\mathbf{2}$ & TFP & Product \\
\hline 1 & 0.1 eq. & 0.1 eq. & $\sim 10 \%$ & PNT 1 \\
2 & 0.2 eq. & 0.2 eq. & $\sim 20 \%$ & PNT 2 \\
3 & 0.3 eq. & 0.3 eq. & $\sim 50 \%$ & PNT 3 \\
4 & 0.4 eq. & 0.4 eq. & $\sim 80 \%$ & PNT 4 \\
5 & 0.5 eq. & 0.5 eq. & $\sim 70 \%$ & PNT 5 \\
\hline
\end{tabular}

In order to improve the tube-forming percentage (TFP), the ratios of tris-amine to MONT were screened. The surface morphologies and TFPs (visual, the same below) of PNTs 1 5 were characterized by SEM images. The results were summarized in Table 1. According to SEM images of PNTs 1 5, we found when the amount of tris-amine was 0.4 molar equivalent of MONT, the highest TFP was obtained. The structures and diameter distributions of PNT 4 were further characterized by STEM. The SEM and STEM images of PNT 4 are shown in Fig. 3. The lengths of PNT 4 are about $500 \mathrm{~nm} \sim 3 \mu \mathrm{m}$, outer diameters are $80 \sim 120 \mathrm{~nm}$ and inner diameters are $60 \sim 100$ nm.
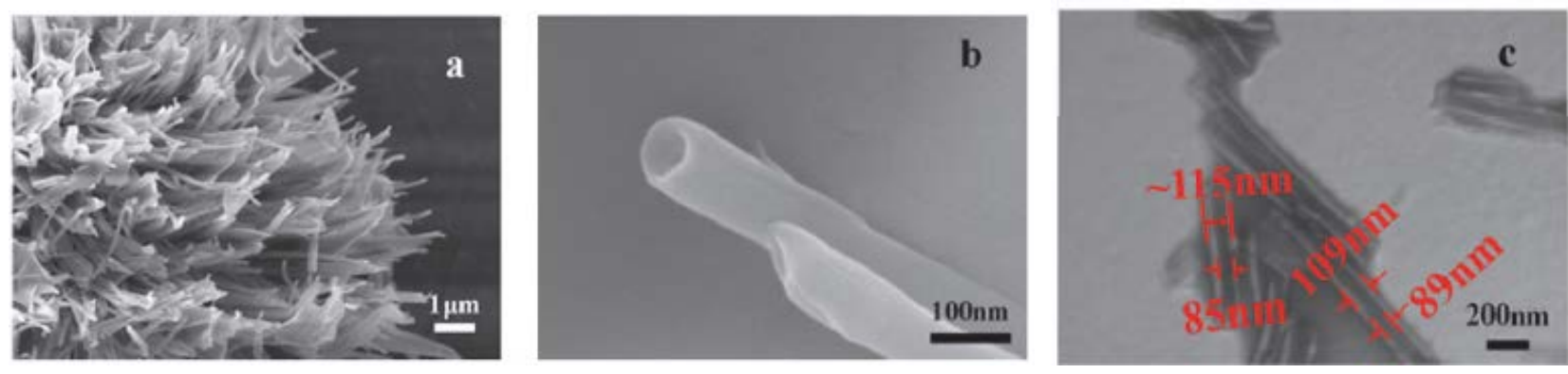

Fig. 3. SEM and STEM images of PNT 4

When the amounts of tris-amine were $0.1 \sim 0.3$ molar equivalent of MONT, TFPs of PNTs $1 \sim 3$ were low, $10 \%, 20 \%$ and $50 \%$ respectively, and a lot of sheet-like structures were found. The inadequate tris-amines sparsely distributed on the surface of the template. Therefore, there should be many large vacant spaces bigger than the size of ester 2, which resulted in many uncoupling areas during cross-linked process. After removal of the internal template, the cross-linking layers would be discontinuous in these uncoupling areas, which is the reason that a lot of sheet-like structures form. With the increases of tris-amine/MONT mole ratio, the TFPs of PNTs gradually increased. When the amount of tris-amine was 0.4 molar equivalent of MONT, up to $80 \%$ cross-linked polymer products formed PNT. At this molar ratio, the tris-amine was able to evenly form moderately wrapped layer on the surface of MONT, and then formed a homogeneous polymer layer surrounding the tube after cross-linked reaction. The PNTs with uniform lengths and diameters could be obtained after removal of the internal template. When the amount of tris-amine was 0.5 molar equivalent of MONT, the TFP slightly decreased to about $70 \%$. The sufficient tris-amine completely wrapped the surface of MONT even freely remained in the solvent. The amino groups of free tris-amine reacted with 2 to generate breakpoints, which reduced the cross-linked probability on the surface of MONT, leading to the decrease of TFP.

For determining the compositions and structures of MONT and PNT, the MONT and PNT were characterized by differential scanning calorimetry (DSC) and X-ray diffraction (XRD). In the DSC curves of MONT, as shown in Fig. 4a, there is a broad peak between $70 \sim 120^{\circ} \mathrm{C}$, which is heat absorption for destroying self-assembled structures, including the van der Waals forces, hydrogen bondings and other intermolecular forces. The peak at $210^{\circ} \mathrm{C}$ probably means the heat decomposition of the copper salt of amphiphilic molecule 1. Further increasing the temperature will 
lead to the carbonization of the decomposed organic compounds, which give peaks higher than 220 ${ }^{\circ} \mathrm{C}$. Different from the MONT, there is no any heat absorption peak in the temperature below $150{ }^{\circ} \mathrm{C}$ in DSC curve of PNT, indicating no self-assembled forces for PNT. With the temperature up to 170 ${ }^{\circ} \mathrm{C}$, the PNT curve displays a high sharp peak because the sample probably occurs to absorb heat to melt. When the temperature rises to $220^{\circ} \mathrm{C}$, the PNT curve shows an absorption peak because of the decomposition even carbonization of polymers. Through the analyses of the DSC curves for MONT and PNT, we confirm that PNT has a higher thermal stability than self-assemblied MONT.
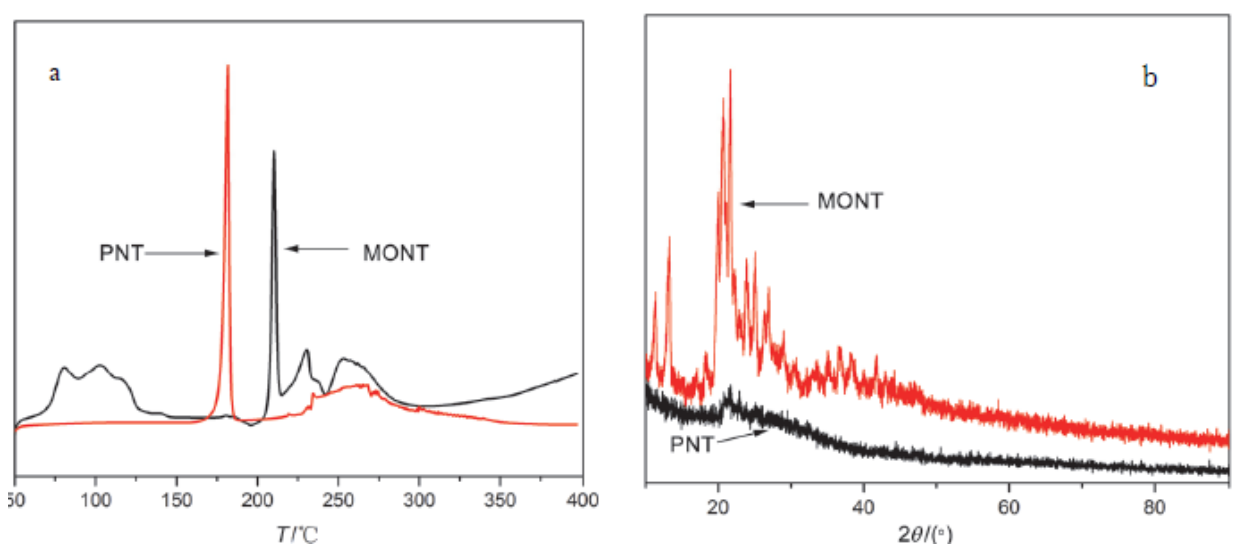

Fig. 4. DSC(a) and XRD(b) curves of MONT and PNT

In the XRD curves of MONT and PNT(shown in Fig. 4b), we easily find the $\mathrm{Cu}^{2+}$ characteristic peaks in MONT curve, which proves the existence of copper ion complexes in MONT. Conversely, only very weak peaks of $\mathrm{Cu}^{2+}$ are able to be observed in PNT curve, indicating most of MONT template successfully removed. The trace of $\mathrm{Cu}^{2+}$ comes from two possible ways: one is a trace of template unsuccessfully removed; the other is a trace of residual carboxyl groups from citric acid combining with copper ions in the process for removing the template.

\section{Conclusion}

We have developed a simple process for the preparation of PNT by using MONT as a template and dendritic amine and acid as precursor molecules. A dendritic amine firstly coordinates with copper ions on the surface of template, and then forms a coating layer on the surface of MONT. The coating layer is further cross-linked by an active ester of dendritic acid. Finally, the self-assembled template is removed and the PNT is successfully obtained. The PNT were characterized by SEM, STEM, DSC and XRD. The results show that when the amount of tris-amine is 0.4 molar equivalent of MONT, up to $80 \%$ cross-linked products form PNT. The lengths of PNTs are about 500nm $3 \mu \mathrm{m}$, inner diameters are $80 \sim 100 \mathrm{~nm}$ and outer diameters are $100 \sim 125 \mathrm{~nm}$. Next, we will further explore the chemical modification of PNT and its application as a drug carrier.

\section{Acknowledgement}

In this paper, the research was sponsored by the Natural Science Foundation of Liaoning Province of China (No. 2015020246) and the Foundation of University of Science and Technology Liaoning (2015TD02)

\section{References}

[1] Fu G D, Li G L, Neoh K G, et al. Hollow polymeric nanostructures-Synthesis, morphology and function[J]. Progress in Polymer Science, 2011, 36(1): 127-167.

[2] Yin Z, Zheng Q. Controlled synthesis and energy applications of one-dimensional conducting polymer nanostructures: an overview[J]. Advanced Energy Materials, 2012, 2(2): 179-218. 
[3] Aida T, Meijer E W, Stupp S I. Functional supramolecular polymers[J]. Science, 2012, 335(6070): 813-817.

[4] Lee J, Kim S M, Lee I S. Functionalization of hollow nanoparticles for nanoreactor applications[J]. Nano Today, 2014, 9(5): 631-667.

[5] Guix M, Mayorga-Martinez C C, Merkoçi A. Nano/micromotors in (bio) chemical science applications[J]. Chemical Reviews, 2014, 114(12): 6285-6322.

[6] Yang L, Tan X, Wang Z, et al. Supramolecular polymers: historical development, preparation, characterization, and functions[J]. Chemical Reviews, 2015, 115(15): 7196-7239.

[7] Stupp S I, Palmer L C. Supramolecular chemistry and self-assembly in organic materials design[J]. Chemistry of Materials, 2013, 26(1): 507-518.

[8] Chapman R, Danial M, Koh M L, et al. Design and properties of functional nanotubes from the self-assembly of cyclic peptide templates[J]. Chemical Society Reviews, 2012, 41(18): 6023-6041.

[9] Wu D, Xu F, Sun B, et al. Design and preparation of porous polymers[J]. Chemical Reviews, 2012, 112(7): 3959-4015.

[10] Liu Y, Goebl J, Yin Y. Templated synthesis of nanostructured materials[J]. Chemical Society Reviews, 2013, 42(7): 2610-2653.

[11] Martin C R. Nanomaterials-a membrane-based synthetic approach[J]. Science, 1994, 266(5193): 1961-1966.

[12] Steinhart M, Wendorff J H, Greiner A, et al. Polymer nanotubes by wetting of ordered porous templates[J]. Science, 2002, 296(5575): 1997.

[13] Zhang J, Li C M. Nanoporous metals: fabrication strategies and advanced electrochemical applications in catalysis, sensing and energy systems[J]. Chemical Society Reviews, 2012, 41(21): 7016-7031.

[14] Panda T, Kundu T, Banerjee R. Self-assembled one dimensional functionalized metal-organic nanotubes (MONTs) for proton conduction[J]. Chemical Communications,2012,48(44): 5464-5466.

[15] Shimizu T, Masuda M, Minamikawa H. Supramolecular nanotube architectures based on amphiphilic molecules[J]. Chemical reviews, 2005, 105(4): 1401-1444.

[16] Shimizu T. Self-assembled organic nanotubes: Toward attoliter chemistry[J]. Journal of Polymer Science Part A: Polymer Chemistry, 2008, 46(8): 2601-2611.

[17] Shimizu T, Minamikawa H, Kogiso M, et al. Self-organized nanotube materials and their application in bioengineering[J]. Polymer Journal, 2014, 46(12): 831-858.

[18] Kameta N. Soft nanotube hosts for capsulation and release of molecules, macromolecules, and nanomaterials[J]. Journal of Inclusion Phenomena and Macrocyclic Chemistry, 2014, 79(1-2): $1-22$.

[19] Kogiso M, Zhou Y, Shimizu T. Instant preparation of self-assembled metal-complexed lipid nanotubes that act as templates to produce metal-oxide nanotubes[J]. Advanced Materials, 2007, 19(2): 242-246.

[20] Mathews A S, Kim I, Ha C S. Synthesis, characterization, and properties of fully aliphatic polyimides and their derivatives for microelectronics and optoelectronics applications[J]. Macromolecular Research, 2007, 15(2): 114-128. 